\title{
L'anaphore rhétorique
}

Figure des figures du discours électoral de Nicolas Sarkozy

Rhetoric Anaphora. The Main Figure of Nicolas Sarkozy's Campaign Speech

\section{Damon Mayaffre}

\section{(2) OpenEdition}

Journals

Édition électronique

URL : http://journals.openedition.org/pratiques/2418

DOI : $10.4000 /$ pratiques. 2418

ISSN : 2425-2042

Éditeur

Centre de recherche sur les médiations (CREM)

Référence électronique

Damon Mayaffre, «L'anaphore rhétorique », Pratiques [En ligne], 165-166 | 2015, mis en ligne le 01 octobre 2015, consulté le 10 décembre 2020. URL : http://journals.openedition.org/pratiques/2418 ; DOI : https://doi.org/10.4000/pratiques.2418

Ce document a été généré automatiquement le 10 décembre 2020.

(c) Tous droits réservés 


\title{
L'anaphore rhétorique
}

Figure des figures du discours électoral de Nicolas Sarkozy

Rhetoric Anaphora. The Main Figure of Nicolas Sarkozy's Campaign Speech

\author{
Damon Mayaffre
}

\section{Introduction}

1 L'anaphore rhétorique est une figure identifiée au moins depuis la Rhétorique à Herennius et pratiquée par tout locuteur politique qui entend convaincre par l'esthétique et l'efficacité de la répétition, comme Démosthène, déjà, selon le traité de Tiberius. Seulement, durant la campagne électorale 2007, Nicolas Sarkozy choisit de faire de l'anaphore rhétorique non pas un usage ponctuel mais un sur-usage systématique, sans doute dans des proportions sans égales dans l'histoire de la parole politique.

2 Nous recenserons ainsi, en quelques mois, presque 10000 reprises anaphoriques dans le corpus exhaustif des discours de campagne du futur président ${ }^{1}$, et nous nous interrogerons sur les conditions de félicité d'un procédé aussi systématique : la figure épouse et construit, idéalement, l'éthos et le programme sarkozystes faits de volontarisme affiché et de convictions déclarées, sur lesquels le candidat sut se faire élire.

3 Une typologie sommaire des anaphores sera alors proposée même s'il est incertain de prétendre épuiser ces 10000 exemples dans une nomenclature finie, en identifiant à chaque fois les effets pragmatiques dans un contexte électoral particulier. Enfin, un zoom sera proposé sur des anaphores remarquables car en apparence complexes voire contradictoires : l'anaphore filée qui rompt de manière problématique la répétition du signifiant en le faisant évoluer ou l'anaphore d'anaphores qui répète non plus un signifiant mais, précisément, le procédé anaphorique (i.e. le phénomène de reprise) luimême.

4 Dans tous les cas, le propos sera de confronter le texte et le contexte, c'est-à-dire la figure rhétorique et la situation discursive électorale, pour comprendre un procédé de 
discours éternel mais toujours actualisé, ici par un locuteur-candidat à la conquête du suffrage universel ${ }^{2}$.

\section{Dix mille anaphores ? Approximation chiffrée}

\subsection{Figure rhétorique et traitement logométrique}

Les figures de style échappent pour la plupart à l'approche automatique et statistique du discours : l'ordinateur reste insensible aux charmes d'une litote et la statistique ignore par définition des fleurs rhétoriques remarquables le plus souvent par leur rareté; en d'autres termes encore, "l'opacité communément reconnue de leur signifiant» (Bonhomme, 2002b) rend les figures difficilement saisissables par une analyse automatique qui a besoin de s'appuyer sur des marques formelles, stables, obvies et récurrentes du corpus ${ }^{3}$.

Pourtant, l'étude logométrique du discours de Sarkozy que nous avons entreprise dans un ouvrage récent (Mayaffre, 2012) met à jour une figure majeure, repérable car fonctionnant sur l'itération : l'anaphore rhétorique, sur laquelle le candidat bâtit une grande part de l'efficacité de son verbe.

7 Présente, sur-présente, omniprésente dans le corpus électoral de Sarkozy, l'anaphore rhétorique reste néanmoins difficilement dénombrable en un chiffrage précis, et nous sommes condamnés à l'approximation.

8 L'étude quantitative des segments répétés (séquences de plusieurs mots répétées dans le corpus $^{4}$ ) que proposent des logiciels comme Lexico ou Hyperbase indique plusieurs dizaines de milliers d'items, parfois longs d'une dizaine de mots, chez Sarkozy en 2007 ; et ce nombre est sans comparaison avec ceux des autres candidats, dans la même situation d'énonciation, auxquels nous avons confronté Sarkozy.

Cependant, si les segments répétés sont un premier indice parlant, ils sous-estiment et sur-estiment le phénomène anaphorique. Sous-estimation d'abord car les segments répétés repèrent les répétitions composées d'au moins deux mots, or l'anaphore peut très bien concerner un mot unique comme dans l'emblématique "Rome... Rome... Rome...» de Corneille. Sur-estimation surtout car toute reprise n'est pas anaphorique notamment lorsque les unités répétées sont trop éloignées dans le texte pour se faire encore écho.

Sur un corpus légèrement différent du nôtre, et avec un mode de calcul plus savant, mais dont il reconnait les limites, J. Véronis conclut quant à lui qu'en 2007, 38 \% des phrases de Sarkozy sont anaphoriques, et ici aussi cette proportion est très supérieure (environ le double) de celle trouvée dans d'autres discours (figure 1). 
Figure 1. Proportion d'anaphores dans le corpus électoral 2007 (selon L.-J. Calvet et J. Véronis, $2007: 34)$

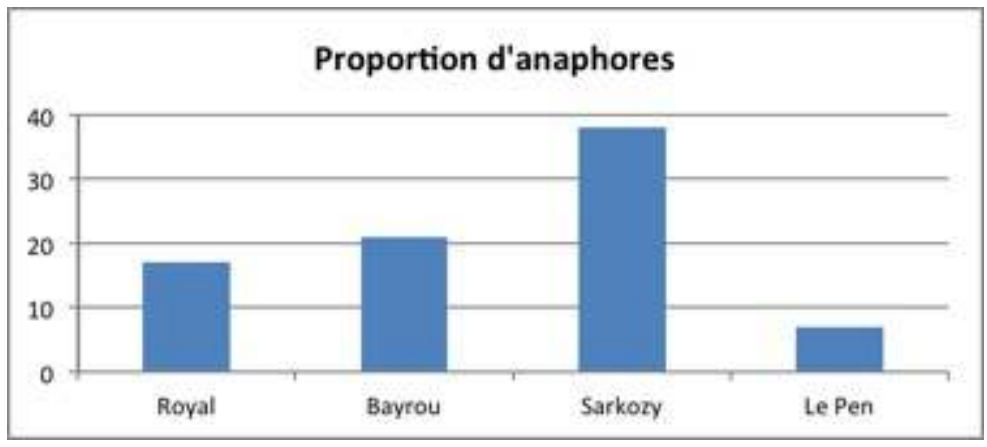

11 Plus d'une phrase sur trois que prononce Sarkozy jouerait donc de l'attaque réitérée. Et ce constat, quoique spectaculaire, ne dit encore rien de l'ampleur du phénomène dans le corpus.

\subsection{L'impossible dénombrement}

Dans son calcul, en effet, J. Véronis s'en tient aux «triplets » (trois mots) répétés, là où nous disions que l'anaphore peut concerner un mot simple, mais surtout il s'en tient aux seules anaphores de début de phrase, là où nous considérons que le phénomène anaphorique peut concerner, de manière beaucoup plus complexe et comme nous l'illustrerons abondamment, des répétitions de milieu ou de fin de phrase (épiphore) ; en position sujet, objet, circonstancielle, etc. (voir infra); syntaxiquement plus ou moins en attaque de proposition ${ }^{5}$.

13 En réalité, dans cette définition large du phénomène anaphorique - tout phénomène explicite de reprise dont on pourra entendre facilement l'écho-, le décompte strict de la figure sur un grand corpus comme le nôtre est impossible, et d'autant plus impossible que Sarkozy s'applique à produire des anaphores redoublées, des anaphores enchâssées, des «répétitions réticulaires" (ici même, E. Prak-Derrington), des anaphores filées dont l'identification et les limites sont incertaines - car relevant de l'interprétation $\mathrm{du}$ récepteur puis de l'analyste - y compris après une exégèse approfondie. Un passage autour de l'anaphore de Sarkozy par laquelle le candidat affirmait avoir changé, illustre la complexité de l'entreprise de décompte :

\section{EXEMPLE 1}

"J'ai changé. J'ai changé parce qu'à l'instant même où vous m'avez désigné j'ai cessé d'être l'homme d'un seul parti, fût-il le premier de France. J'ai changé parce que l'élection présidentielle est une épreuve de vérité à laquelle nul ne peut se soustraire. Parce que cette vérité je vous la dois. Parce que cette vérité je la dois aux Français.

J'ai changé parce que les épreuves de la vie m'ont changé. Je veux le dire avec pudeur mais je veux le dire parce que c'est la vérité et parce qu'on ne peut pas comprendre la peine de l'autre si on ne l'a pas éprouvée soi-même.

[...] J'ai changé parce que le pouvoir m'a changé. Parce qu'il m'a fait ressentir l'écrasante responsabilité morale de la politique.

J'ai changé parce que nul ne peut rester le même devant le visage accablé des parents d'une jeune fille brûlée vive. Parce que nul ne peut rester le même devant la douleur qu'éprouve le mari d'une jeune femme tuée par un multirécidiviste condamné 10 fois pour violences et déjà une fois pour meurtre.

J'ai changé quand j'ai visité le mémorial de Yad Vashem dédié aux victimes de la 
Shoah.

[...] J'ai changé quand j'ai lu à Tibhirine le testament bouleversant de frère Christian, enlevé puis égorgé par des fanatiques avec 6 autres moines de son monastère. Le GIA avait prévenu : "nous égorgerons". On retrouva les 7 têtes des moines suppliciés sans leurs corps.

J'ai changé quand j'ai rencontré Mandel, ce grand Français. J'avais voulu écrire sa vie pour réparer une injustice, pour changer le regard des autres sur cette destinée tragique. C'est mon regard sur la politique qui s'en est trouvé transformé » (N. Sarkozy, 14 janvier 2007, Paris).

14 Si le segment anaphorique stricto sensu «j'ai changé » peut être facilement compté, faut-il distinguer, c'est-à-dire compter séparément, et compter à nouveau, les «j'ai changé quand... » et les «j'ai changé parce que...» ? De fait, le " parce que » parait très vite comme une des clés anaphoriques principales du passage ( 11 fois scandé) et prend son indépendance et se déploie sans le «j'ai changé » initial. Mais alors, il conviendrait de décomposer à son tour le "parce que » en deux anaphores au moins secondaires : «parce que cette vérité..., parce que cette vérité... » et " parce que nul ne peut... parce que nul ne peut... ». Enfin en dernière extrémité, on peut remarquer que ces deux dernières formules, construites respectivement autour de la "vérité » ou de "nul » reprennent deux mots que l'on retrouve répétés indépendamment, ailleurs dans le passage, comme pour augmenter l'écho ou la symphonie anaphorique.

Au final, c'est donc à une approximation chiffrée grâce aux segments répétés d'un côté et au repérage manuel par sondage de l'autre que nous nous sommes livré pour conclure que le corpus Sarkozy compte sans doute près de 10000 séquences relevant d'une anaphore rhétorique; et cette approximation retrouve et généralise la conclusion de J. Véronis. Ce n'est pas simplement une phrase sur trois qui commence par une attaque anaphorique, mais environ un mot sur cinq du corpus qui est contenu dans une structure anaphorique ${ }^{6}$. Ce nombre et cette concentration n'ont jamais été rencontrés dans aucun des corpus politiques, nombreux, jusqu'ici étudiés en logométrie. L'anaphore rhétorique participe à l'esthétique systématique du verbe électoral sarkozyste. Elle lui donne une efficacité qu'il reste à décrire et participe, avec des formules inlassablement martelées, à la sloganisation des discours de tribune relayés par la télévision.

\section{Généralités : brève typologie et approche pragmatique de l'anaphore chez Sarkozy}

16 Les anaphores rhétoriques sont si nombreuses qu'il conviendrait de déployer plusieurs typologies pour prétendre les épuiser. M. Charolles, à propos de l'anaphore en général, conclut à l'impossibilité d'une classification (1991), et nous nous contenterons, à propos de l'anaphore rhétorique en particulier, de quelques départages, le plus souvent dichotomiques.

\subsection{Anaphore nominale versus anaphore verbale}

La distinction la plus évidente est celle qui sépare grossièrement les anaphores d'essence nominale et celles d'essence verbale: elles participent, à nos yeux, de deux pragmatiques du discours distinctes. 
L'anaphore nominale n'est pas la plus présente dans le corpus; mais elle est la plus classique en rhétorique et compte tout de même plusieurs milliers d'exemples chez Sarkozy. Elle consiste, d'une manière ou d'une autre, en la répétition d'un substantif fort (le «travail », la « France », la « délinquance », l'« éducation », le « pays », etc.). Dans le discours politique, elle a une dimension programmatique, axiologique ou idéologique grâce à la manipulation de concepts-clés que Sarkozy va définir, promouvoir ou pourfendre - et en tout cas répéter. L'exemple suivant entre mille est éloquent dans la mesure où Sarkozy, comme souvent, introduit le signifiant qu'il va anaphoriser :

\section{EXEMPLE 2}

«Le mot autorité ne me fait pas peur, le mot morale non plus. L'autorité ce n'est pas l'autoritarisme. L'autorité ce n'est pas l'obéissance aveugle. C'est l'obéissance consentie. L'autorité ce n'est pas la crainte. C'est le respect. L'autorité ce n'est pas la force, c'est la légitimité. [...] L'autorité, c'est quand le maître est respecté, c'est quand la loi est la même pour tous, quand le crime est puni et le mérite récompensé... » (N. Sarkozy, 23 février 2007, Perpignan).

La mécanique est explicitée par l'orateur lui-même. Parce que le " mot autorité » serait devenu tabou, Nicolas Sarkozy va, à contresens de la pensée unique, l'utiliser, le répéter, le marteler. À minima, dans les termes de la communication, il s'agit explicitement d'imposer par la répétition l'agenda setting du discours et de la campagne électorale; et de fait, les observateurs ont pu constater que Sarkozy avait su dicter le rythme et les thématiques médiatiques du moment. Plus profondément, et très rapidement dans le discours, en passant du mot à la valeur, Sarkozy peut développer, définir et à nouveau imposer, un concept, un programme ou une axiologie. Et l'on dira avec Marc Bonhomme (2005: 196) que «la figure donne d'emblée une forte présence conceptuelle au thème abordé (fonction cognitive de soulignement), tout en frappant le lecteur, en attaque du texte (fonction phatique de contact)». Comme suggéré dans la citation, Sarkozy fera de même avec la «morale » puis ailleurs avec d'autres motsvaleurs intéressants du discours politique : le «travail », le « mérite », la «famille», le " capitalisme ", etc.

Les anaphores verbales quant à elles, portées si possible par la première personne du singulier et construites le plus souvent autour d'un verbe fort, ont une autre fonction : elles ont pour vocation de construire l'éthos charismatique du candidat, qui exhibe son ambition voire son entêtement par une posture énonciative sourde, ostensive, assumée. La plus célèbre, car parmi les plus longues, fut le "je veux être le président qui... » quelque 27 fois répété de suite à la tribune, et à côté de laquelle le « moi, président de la République » de Hollande durant le débat télévisé de 2012 - un véritable détournement 5 ans après - paraitra dérisoire (11 fois seulement).

\section{EXEMPLE 3}

«Je veux être le Président de l'augmentation du pouvoir d'achat. Je veux être celui qui vous garantit que si vous travaillez plus, si vous prenez plus de risque, si vous vous engagez plus, vous gagnerez davantage. Je veux être le Président du peuple qui a bien compris que les RTT ne servent à rien si on n'a pas de quoi payer des vacances à ses enfants. [...] Je veux être le Président de tous ces Français qui pensent que l'assistanat est dégradant pour la personne humaine. Je veux être le Président qui s'efforcera de moraliser le capitalisme. [...] Je veux être le Président qui va remettre la morale au cœur de la politique...[etc.] 》 (N. Sarkozy, 14 janvier 2007, Paris). 
21 se factorisent. Le volontarisme sarkozyste s'exprime par la multiplication du lexique (vouloir, je) et de la rhétorique (la scansion anaphorique) : pour le moins nous avons affaire ici à un candidat déterminé.

Le « je veux... » exhibé de ce passage paraitra caricatural, mais le martèlement des « j’ai changé... » de l'exemple 1 ou celui des «je rêve... » de l'exemple à venir participent, nous semble-t-il, de cette même construction charismatique d'un chef qui sait ce qu'il pense, dit, veut, désire :

\section{EXEMPLE 4}

"Je rêve d'une France qui se lève tout entière pour dire "non" au renoncement, "non" à la fatalité, "non" au déclin.

Je rêve d'une France qui se lève tout entière pour dire "non" à tous ceux qui dans les cercles du pouvoir politique, administratif, économique, culturel, syndical sont convaincus que la France ne signifie plus rien, qu'elle est un vestige du passé, qu'elle a si peu d'existence qu'elle n'a même plus d'identité et qui considèrent qu'ils ne lui doivent plus rien.

Je rêve d'une France qui se lève tout entière pour dire "non" à ceux qui n'envisagent pour elle qu'un destin médiocre et un rôle subalterne.

Je rêve d'une France qui se lève tout entière pour dire "non" parce qu'elle croit encore en elle, parce qu'elle croit encore qu'elle peut agir, qu'elle peut créer, qu'elle peut être grande et qu'elle n'est pas condamnée à subir » (N. Sarkozy, 10 avril 2007, Tours).

Si l'onirique pourra être ressenti comme moins fort que le volontif, le charisme de Sarkozy bénéficie en pareil cas de celui de Martin Luther King duquel le candidat de 2007 n'hésite pas à se revendiquer ${ }^{7}$; nous verrons infra que cette charisma et l'autorité qui en découle sont souvent favorisées par la négation et, dans ce passage, par ce « non » qui renvoie, à côté de Luther King, au général de Gaulle.

Enfin le pronom personnel de première personne («je veux... », «j’ai changé... », « je rêve...", etc.), est sans doute pour beaucoup, pensera-t-on, dans l'effet ressenti. Pourtant des formules impersonnelles sur le mode du « il faut..., il faut..., il faut... » ou «ce n'est plus possible..., ce n'est plus possible..., ce n'est plus possible...» (cf. exemple 9 , infra) conspirent à chaque fois au même résultat ${ }^{8}$.

\subsection{Anaphore affirmative, négative, interrogative}

Les exemples cités jusqu'ici relèvent pour la plupart d'anaphores affirmatives, mais il est remarquable de constater que Sarkozy s'applique à conjuguer ses anaphores aussi sous la forme interrogative ou négative; et encore interro-négative et exclamative. Comme par jeu, le locuteur et ses plumes prennent un soin particulier à explorer toutes les possibilités que leur offre la langue en la matière. L'anaphore interrogative doit être illustrée car sa fonction pragmatique est particulière et très significative du discours sarkozyste. Avec ses 46 répétitions - dont on ne pourra reproduire qu'un court extrait - l'anaphore suivante est la plus prodigieuse du corpus :

\section{EXEMPLE 5}

« Alors, pourquoi tant d'attaques personnelles, pourquoi tant de violence, pourquoi tant de haine? Oui, pourquoi tant de haine? Parce que je parle de la France? De son identité ? De ses valeurs ? [...] Pourquoi tant de haine ? Parce que je dis que dans l'identité française il y a des valeurs qui ne sont pas négociables ? [...] Pourquoi tant de haine ? Parce que je n'accepte pas la repentance? [...] Parce que je considère que 
la France n'a pas à avoir honte de son histoire ? Pourquoi tant de haine ? Parce que je dis que tous les Français n'étaient pas pétainistes? [...] Pourquoi tant de haine? Parce que je dis que tous les colons n'étaient pas des exploiteurs? [...] Pourquoi tant de haine? Parce que je dis que si la France a une dette morale c'est envers eux, auxquels un jour on n'a donné le choix qu'entre la valise et le cercueil et qui ont tout perdu? [...] Pourquoi tant de haine ? Parce que je dis tout haut ce que tout le monde pense tout bas, que si l'on n'aime pas la France on n'est pas obligé d'y venir et on n'est pas obligé d'y rester [...] Parce que je dis que j'admire Jean-Paul II pour son courage, pour sa fermeté, pour le rôle qu'il a joué dans la fin du communisme en Europe, pour sa profonde spiritualité ? Pourquoi tant de haine? Parce que je dis cette vérité que nous sommes les héritiers de deux mille ans de chrétienté dont les valeurs ont été incorporées dans notre morale laïque ? [...] Pourquoi tant de haine? Parce que je dis que je veux tourner la page de mai 68 ? Parce que je dis que je veux en finir avec l'héritage de mai 68 , avec le relativisme intellectuel et moral de mai 68 ? [...] Pourquoi tant de haine ? Parce que j'appelle voyou un voyou ? Parce qu'un jour j'ai traité des voyous de racailles ? [...] Pourquoi tant de haine ? [...] Parce que je ne veux plus qu'une minorité puisse prendre la majorité des citoyens en otage ? Parce que je ne confonds pas le droit de grève avec le droit de bloquer tout le pays?» (N. Sarkozy, 23 avril 2007, Dijon).

L'interrogation est une des plus grandes caractéristiques statistiques du discours de Sarkozy (Mayaffre, 2012: 236-261). Ici comme ailleurs, l'interrogation permet un retournement de la charge du discours : d'interrogé, Sarkozy se fait interrogateur en même temps que de coupable - car Sarkozy n'a jamais été tendre avec les autres - il devient victime. À la fois avocat et procureur, c'est lui en tout cas, dans un retournement des rôles, qui pose les questions là où l'homme politique se doit habituellement de rendre des comptes. La force anaphorique donne à ces interrogations l'allure du réquisitoire. Normalement soumis au feu des questions auxquelles il se doit de répondre, l'homme politique ici contre-interroge. De plus, l'avalanche de questions - dans ce passage plus d'une centaine de suite!- laisse l'auditoire devant un abime de perplexité, dont seul le locuteur semble détenir l'issue. D'ailleurs, l'anaphore rhétorique interrogative s'enchaine le plus souvent dans le discours avec une anaphore affirmative sur le mode du "parce que »". Pourquoi...? Pourquoi... ? Pourquoi... ? / Parce que... Parce que... Parce que... : c'est à nouveau le charisme de Sarkozy qui se trouve renforcé. L'homme détient à la fois le savoir omniscient et le pouvoir inquisitorial. Il a la force et la légitimité des convictions mais aussi celle de l'interrogation. En tribun d'un peuple victime à travers sa personne de la « haine » de la pensée unique, Sarkozy interpelle, questionne, répond ad libitum dans ses discours et domine la totalité de l'échange.

L'anaphore négative relève au fond du même procédé. Par sa capacité à dire «non ", à dire «ne... pas », Sarkozy s'établit en chef, voire en père sévère : il s'agit à nouveau d'une forte signature statistique de Nicolas Sarkozy indépendamment des anaphores rhétoriques (Mayaffre, 2012 : 304-309). L'exemple 2 n'a pas à être reproduit. L'autorité y est définie par l'affirmative bien sûr («l'autorité c'est..., l'autorité c'est..., l'autorité c'est... ») mais aussi par la négative ( "l'autorité ce n'est pas..., l'autorité ce n'est pas..., l'autorité ce n'est pas... »). Selon H. Nolke, et contre O. Ducrot, la négation a toujours une dimension polémique ${ }^{10}$ dont Sarkozy sait user et abuser : une phrase sur trois chez Sarkozy est négative dans son discours entre 2007 et 2012 (lorsque par exemple une phrase sur dix l'est chez de Gaulle). Et Sarkozy polémique, par la négation de la position de l'autre, avec d'autant plus de force qu'il le fait ici par la répétition anaphorique. L'exemple 4 est tout aussi parlant - ne serait-ce que par la longueur de la séquence 
répétée de 13 mots - de ce discours d'autorité assumée que l'anaphore négative renforce : "Je rêve d'une France qui se lève tout entière pour dire "non"... ». Où l'on remarquera à nouveau l'explicitation du mécanisme : c'est le fait de dire " non » - ou plutôt de le répéter ! - qui devient programme, image, autorité politiques.

\subsection{Anaphore et épiphore}

Questions et réponses, affirmations et négations : le discours de Sarkozy en 2007 est un monument rhétorique dont nous avons montré ailleurs, aussi, les saillances lexicales ou grammaticales remarquables qui le distinguent par exemple du discours sans relief de son prédécesseur Jacques Chirac à l'Elysée.

Les milliers d'anaphores, en tête de phrase et en position sujet, servent à Nicolas Sarkozy à conditionner l'auditoire par une amorce fixement martelée 5, 10, 20, 30, 46 fois de suite. Mais Nicolas Sarkozy va plus loin dans la stratégie rhétorique et triomphe définitivement de l'esprit de l'auditoire dans des passages plus forts encore, ou plus contraints/contraignants qui combinent anaphore rhétorique et épiphore rhétorique.

\section{EXEMPLE 6}

"Vous en avez assez de la langue de bois et vous avez raison!

Vous en avez assez de la pensée unique et vous avez raison!» (N. Sarkozy, 20 mars 2007, Villebon-sur-Yvette)

Ou :

\section{EXEMPLE 7}

«On vous a souvent trompés. Je le sais.

On vous a parfois menti. Je le sais.

On vous a même trahis. Je le sais.

Et pourtant vous êtes là !

Vous êtes là, parce que la France pour vous ce n'est pas fini !

Vous êtes là, parce que la politique pour vous ce n'est pas fini !» (ibid.)

L'espace de liberté du discours, c'est-à-dire celui que le locuteur négocie avec l'auditoire, se trouve fermement borné, phrase après phrase, en amont et en aval. À ce stade, il n'est sans doute pas exagéré de parler d'une sloganisation du discours et de sa dimension propagandique. C'est ce procédé (anaphore en entrée, épiphore en sortie), proche du "bourrage de crâne", que Sarkozy utilise dans ses passages les plus dissensuels, à visées polémiques et idéologiques. C'est particulièrement le cas lorsqu'il fixe, dans une formulation catégorique, comme objectif à son élection de «liquider l'héritage de mai $1968 »^{11}$ et qu'il revient dans les charges les plus violentes du corpus sur la France qui aurait été avilie par les Soixante-huitards :

\section{EXEMPLE 8}

« Mai 68 est hélas passé par là. À bas l'autorité ! C'était cela le programme de mai 68. À bas l'autorité ! Le moment était venu de vivre sans contrainte et de jouir sans entrave. À bas l'autorité ! C'était, prétendaient-ils, la condition de la libération de l'homme aliéné par le travail, par la vie en société, par l'économie, par son éducation et même par sa famille. À bas l'autorité ! Cela voulait dire :

L'obéissance de l'enfant à ses parents, c'est fini ! Démodé ! La supériorité du maître sur l'élève, c'est fini ! Ringard! La soumission à la loi, c'est fini ! Dépassé ! Le pouvoir de police, c'est fini ! Enfin ! Le respect de l'Etat et de ceux qui le représentent, c'est fini ! L'amour de la patrie, la fidélité à la France, à son drapeau, la gratitude vis-à-vis de ceux qui se sont battus pour elle, c'est fini ! La morale, c'est fini ! L'humilité devant le savoir, devant les grandes œuvres de l'esprit humain, c'est fini ! La hiérarchie des valeurs, c'est fini ! La politesse, la courtoisie, le respect 
pour la personne âgée, pour la femme! C'est fini! À bas l'autorité ! Cela voulait dire :

Désormais tout se vaut. Le bien comme le mal, la grandeur comme la bassesse, le vrai comme le faux, le beau comme le laid. Tout se vaut: la parole de l'élève vaut celle de l'instituteur. Une émission de variétés vaut une pièce de Racine. L'intérêt particulier vaut l'intérêt général. Le délinquant vaut la victime. La loi des bandes vaut celle de la République. Le non travail vaut autant que le travail » (N. Sarkozy, 7 février 2007, Toulon).

D'un point de vue formel, ce passage extraordinaire nous intéresse car il pointe les limites ou l'extension de l'anaphore. Très contraint par des structures anaphoriques d'abord («À bas l'autorité !») puis épiphorique («C'est fini!»), qui laissent sur l'auditeur une impression indélébile (ne serait-ce que par leur tournure exclamative), le passage se termine par la simple répétition du verbe "valoir » à la troisième personne. À strictement parler, cette répétition ne sera pas qualifiée d'anaphore par les rhétoriciens. Pourtant le co-texte (i.e. l'ensemble du passage, sinon du discours) détermine l'interprétation, comme le global détermine le local, et l'on ressentira ce martèlement comme anaphorique à la suite des anaphores ou épiphores qui le précèdent. Comme le pointe G. Salvan (2012-2013b: 8, 9), citant M. Monte puis M. Bonhomme, les figures sont d'abord une "décision interprétative ", c'est-à-dire aussi, dans une perspective herméneutique, des «constructions contextuelles». En l'occurrence, Nicolas Sarkozy force l'interprétation puisque qu'outre les anaphores et épiphores précédentes qui le contextualisent ou le déterminent, le "vaut " succède à une pseudo-anaphore rhétorique, avortée mais visible, dont il devient l'artefact ou la réduction : « Désormais tout se vaut..., tout se vaut..., vaut..., vaut..., vaut... ».

\section{Raffinement : anaphores cataphoriques et anaphores d'anaphores}

\subsection{L'anaphore cataphorique}

Nous nommons, par ironie, anaphore cataphorique, les anaphores rhétoriques dont la structure relève de la cataphore grammaticale. La plus remarquable est sans doute celle construite à partir de l'affirmation-négation «ça ne peut plus durer... " répétée à l'initiale, suivie en deuxième partie de phrase de son conséquent.

\section{EXEMPLE 9}

« Ici, à Toulon, comme partout en France, j'entends ce cri qui s'élève des profondeurs du pays et dont l'écho semble se répéter indéfiniment : "ça ne peut plus durer".

Ça ne peut plus durer les travailleurs pauvres qui se demandent quoi faire pour s'en sortir.

Ça ne peut plus durer le pouvoir d'achat trop faible, les enfants condamnés à vivre moins bien que leurs parents, la difficulté de se loger décemment.

Ça ne peut plus durer l'assisté qui gagne plus que le travailleur, l'école qui n'apprend pas à lire, à écrire, à compter correctement.

Ça ne peut plus durer la violence des multi-récidivistes.

Ça ne peut plus durer le petit voyou et le patron voyou qui restent impunis.

Ça ne peut plus durer les parachutes en or pour celui qui échoue, les stock options réservées à quelques-uns.

Ça ne peut plus durer les femmes moins payées que les hommes, les mariages forcés, la loi des grands frères, la polygamie, l'excision.

Ça ne peut plus durer les délocalisations provoquées par la concurrence déloyale, le 
dumping social, écologique, fiscal.

Ça ne peut plus durer le travail découragé, le travailleur démoralisé.

Ça ne peut plus durer le travailleur qui paye pour tout le monde.

Ça ne peut plus durer les valeurs de la France bafouée, l'histoire de France répudiée,

la nation dénigrée.

Ça ne peut plus durer la France qu'on regarde se défaire en proclamant que l'on n'y

peut rien » (N. Sarkozy, 7 février 2007, Toulon).

L'anaphore que nous qualifierons de filée, enfin, permet de conclure. Par exemple :

\section{EXEMPLE 10}

«La France c'est le nom de notre destin commun.

La France c'est une solidarité.

La France c'est une identité.

La France c'est une culture.

La France c'est un projet collectif, c'est la volonté de vivre ensemble et de construire ensemble.

[...] La France c'est le pays qui le premier a proclamé que c'était le bonheur et non la volonté de puissance qui devait être la fin de toute politique.

La France c'est le pays qui s'est le plus battu pour la liberté.

C'est le pays qui a enseigné aux hommes l'égalité des droits.

C'est le pays qui en hissant la fraternité au même niveau que la liberté et l'égalité a dit à tous les hommes comme Antigone : "Je suis née pour partager l'amour, non pour partager la haine". 
La France c'est notre pays. Nous n'en avons pas d'autre.

Le mépriser ce serait nous mépriser nous-mêmes.

Le haïr ce serait nous haïr nous-mêmes.

La fierté d'être français c'est ce qui nous unit par-delà nos différences.

La fierté d'être français c'est ce que nous voulons partager avec ceux qui veulent

devenir français » (N. Sarkozy, 10 avril 2007, Tours).

Par définition, l'anaphore est la répétition du même signifiant; et il est facile de repérer les différentes répétitions du passage d'autant qu'elles sont clairement situées en début de phrase. Pourtant ici, l'on comprend derrière les refrains anaphoriques des signifiants que ce qui est répété, c'est le signifié. Entre rupture (du signifiant) et continuité (de la figure rhétorique), c'est bien le patriotisme qui est animé: «la France..., la France... », « le pays... le pays... », « la fierté d'être français..., la fierté d'être français... $»^{14}$. En combinant structures anaphoriques indentifiables et reprises isotopiques, c'est-à-dire en filant ses anaphores rhétoriques autour de signifiants quasi synonymes et d'un signifié unique comme le patriotisme, Sarkozy donne une puissance rare à son discours. Cette déclinaison du même (anaphore évolutive ou anaphore filée, en tout cas avec rupture du signifiant) avait été déjà constatée dans des exemples plus faciles comme ceux où Sarkozy passe de l'affirmation à la négation («l'autorité c'est..., l'autorité ce n'est pas... ») ou ceux où Sarkozy fait varier sa figure («j'ai changé parce que..., j'ai changé quand... »), etc.

41 Plus loin encore, les exemples nombreux que nous avons cités tout au long de cette contribution (notamment l'exemple 1) montrent que ce qui est en jeu dans le corpus, ce n'est parfois ni la reprise du signifiant (anaphore stricto sensu), ni la reprise du signifié (anaphore que l'on qualifiera de filée), mais le procédé anaphorique lui-même. Ce qui est inlassablement répété dans le discours et ce que reconnait l'auditeur chez l'orateur Sarkozy, c'est cette figure, véritable matrice du discours, qui revient sans cesse, et donne un rythme envoûtant au parler du candidat: Sarkozy invente l'anaphore d'anaphores.

\section{Conclusion}

42 À l'image de certaines comptines - dont l'orthographe rappelle le sens - le discours de Nicolas Sarkozy ressemble à une antienne que l'orateur psalmodie jusqu'à la fascination de l'auditoire; car qui ne serait pas fasciné par un orateur aussi habité par des affirmations qu'il peut répéter au-delà de toutes les conventions tacites du discours politique ${ }^{15}$ Indéniablement, le procédé anaphorique a une "dimension pathémique » (Bonhomme, 2005 : 173), susceptible de frapper l'esprit de l'auditoire, dont Sarkozy sait jouer.

D'anaphores en anaphores - des milliers dans le corpus - Nicolas Sarkozy construit ainsi son discours par un système de reprise ou de rebond qui ne cherche pas à s'excuser, et que l'on schématisera ainsi en combinant anaphore et anadiplose ${ }^{16}$ : A c'est B, A c'est C, A c'est D. D c'est E, D c'est F, D c'est G. G c'est H, G c'est I, G c'est J. Et ainsi de suite.

L'anaphore systématique et l'anaphore d'anaphores donnent un rythme, une continuité et une cohésion sans égales au corpus électoral de Sarkozy. Le discours est linguistiquement fortement articulé voire "enchainé", et les chevilles de cette articulation sinon les fers de cette chaine, ne sauraient échapper à l'auditoire puisqu'ils 
sont délibérément ostensifs; si nécessaire, du reste, Sarkozy les sur-surlignera par un commentaire métadiscursif ("le mot "autorité" ne me fait pas peur... », «j'entends ce cri qui s'élève des profondeurs du pays et dont l'écho semble se répéter indéfiniment ...", « je rêve d'une France qui dit "non"... », etc.).

Le procédé est habile parce que, outre le fait que l'anaphore donne de la force à un locuteur qui affiche l'entêtement phraséologique, la conviction répétée, la détermination comme les attributs du chef, elle donne une impression de cohérence à un discours qui n'en a pas toujours et le sentiment de progression, par accumulation, qu'il aimerait avoir. De manière critique même, il faut parfois conclure que la figure, évidemment cohésive puisqu'elle donne une cohésion formelle au discours, est là pour suggérer ou forcer une cohérence sémantique et idéologique qui pose problème. L'exemple 9 notamment a montré comment la répétition du même, en amorce, permet en réalité de compiler le différent en fin de phrase, avec des idées qui idéologiquement en France ne relèvent pas de la même analyse, ni du même parti pris (par exemple, la polygamie et les stock-options). L'identique (c'est-à-dire le répété) ici n'est que de surface et a pour vocation de masquer l'hétérogène ; la progression n'est qu'apparente dans un discours bégayé, non hiérarchisé et qui tourne en rond. Et pratiquement toutes les longues anaphores de Sarkozy (« je rêve de X..., je rêve de Y..., je rêve de $Z$ » ou « je veux être le président qui A..., je veux être le président qui B..., je veux être le président qui $C$ ») ont cette fonction de mettre sur un plan d'équivalence des éléments prédicatifs disparates voire contradictoires ( $\mathrm{X}, \mathrm{Y}$ et $\mathrm{Z}, \mathrm{A}, \mathrm{B}$ et $\mathrm{C}$ ), produisant un chaos idéologique qui met K.O. l'auditoire, et dont ne ressort finalement que la performance litanique ou incantatoire d'un leader qui se performe en leader.

Ainsi les anaphores rhétoriques chez Nicolas Sarkozy, comme sans doute toutes les figures, jouent, à l'interface, avec la cohésion et la cohérence du discours, le signifiant et le signifié, la forme et le contenu pour faire entendre beaucoup plus que ce qu'elles disent ; l'électorat, inégalement disposé, les interprètera plus (en 2007) ou moins (en 2012) favorablement.

\section{BIBLIOGRAPHIE}

BONHOMme, M. (dir.) (2002a) : « Figures du discours et ambiguïté », Semen, 15, (en ligne : http:// semen.revues.org/743, consulté le 01/02/14).

- (2002b) : « Présentation », Sémen, 15 (en ligne : http://semen.revues.org/2366, consulté le $01 / 02 / 14)$.

- (2005) : Pragmatique des figures du discours, Paris, H. Champion.

CALAS, F. et al. (2012) : Les Figures à l'épreuve du discours, Paris, Presses de l'université ParisSorbonne.

CALVET, L.-J. \& VÉRonIS, J. (2007) : Les Mots de Nicolas Sarkozy, Paris, Éd. Le Seuil.

CHAROLLES, M. (1991) : «L'anaphore : problèmes de définition et de classification », Verbum, XIV, p. 203-215. 
GARDES TAMINE, J. (2011) : Pour une nouvelle théorie des figures, Paris, Presses universitaires de France.

JAUBERT, A. \& MAYAFFRE, D. (2013) : « Éthos préalable et éthos (re)construit. La transformation de l'humour légendaire de François Hollande », Langage et Société, 146, décembre, p. 71-88.

KOKELBERG, J. (1991) : Les Techniques du style, Paris, Nathan.

MAYAFFRE, D. (2012) : Mesure et démesure du discours, Nicolas Sarkozy (2007-2012), Paris, Presses de Science Po.

- (2013) : «Sarkozysme et populisme. Approche logométrique du discours de Nicolas Sarkozy (2007-2012) », Mots, 103, p. 73-87.

- (2014) : « "Ça suffit comme ça !". La fausse opposition quantitatif / qualitatif à l'épreuve du discours sarkozyste », Corela, hors-série 15 (en ligne : http://corela.revues.org/3543, consulté le 24/03/14).

NøLKE, H. (1992) : « Ne... pas : négation descriptive ou polémique », Langue française, 94, p. 48-67.

PERELMAN C. \& OLBRECHTS-TYTECA, L. (2009) : Traité de l'argumentation, Bruxelles, Éd. de l'Université de Bruxelles.

SALEM, A. (1987) : Pratique des segments répétés. Essai de statistique textuelle, Paris, Klincksieck.

SALVAN, G. (dir.) (2012-2013a) : « Figures et contexte(s) », Le Discours et la langue, t. 4.2.

- (212-2013b) : «Introduction », Le Discours et la langue, t. 4.2, p. 7-14.

TAGUIEFF, P.-A. (2007) : L'Illusion populiste. Essai sur les démagogies de l'âge démocratique, Paris, Flammarion.

\section{NOTES}

1. Le corpus saisi et étudié est composé de la totalité des discours de meeting de Nicolas Sarkozy prononcés en France entre décembre 2006 et mai 2007, soit 34 discours de tribune, représentant 242265 mots-occurrences. Le cas échéant, ces discours seront comparés aux discours des principaux adversaires de Nicolas Sarkozy au premier et second tour de l'élection: Arlette Laguiller, Marie-Georges Buffet, Ségolène Royal, François Bayrou et Jean-Marie Le Pen.

2. Remerciements à Anna Jaubert et Sylvie Mellet pour leurs relectures et suggestions.

3. Sur les vertus mais aussi les limites de l'analyse automatique du discours, voir par exemple A. Jaubert et D. Mayaffre (2013).

4. Les segments répétés sont un des outils-phares de la lexicométrie (Salem, 1987). Suites contigües de formes répétées dans le corpus, ces segments répertorient automatiquement évidemment les mots composés (pomme de terre) ou les collocations (pluie battante) mais aussi les syntagmes semifigés ou des éléments phraséologiques à partir du moment où ils sont récurrents.

5. Nous touchons là à une difficulté définitionnelle de l'anaphore. La plupart des auteurs concède en effet que l'anaphore ne peut être réduite aux répétitions de début de phrase, ni même de proposition. Mais par facilité didactique, ils se rabattent le plus souvent sur des exemples ad hoc. M. Bonhomme (2005: 65) note à propos des figures de répétition: «Du fait de sa plus forte saillance à l'attaque des séquences syntaxiques, l'anaphore rhétorique tend chez certains commentateurs (Kokelberg 1991...) à apparaître comme le prototype de cette famille de figures et à les dénommer génériquement ». C'est précisément dans l'acception élargie ou générique, donnée par exemple par J. Kokelberg (1991), que nous considérons dans cette contribution l'anaphore rhétorique. 
6. Approximation toujours : les anaphores de Sarkozy sont faites, en moyenne, de la reprise de 5 mots. Avec 10000 séquences anaphoriques, ce sont donc 50000 mots qui sont concernés dans un corpus long de 250000 mots, soit 1 mot sur 5 .

7. De manière explicite, par exemple: «Souvenez-vous de Martin Luther King, ce pasteur noir qui a dit un jour à l'Amérique devant le mémorial de Lincoln à Washington : "Je rêve qu'un jour notre pays se lèvera." " (N. Sarkozy, 18 mars 2007, Paris).

8. $\mathrm{Du}$ reste, l'effet charismatique de l'anaphore rhétorique dépasse l'anaphore verbale. L'anaphore nominale précédente sur l'autorité (exemple 2) dont nous avons souligné l'aspect programmatique ou idéologique, avait elle aussi une dimension charismatique : son introduction - «le mot autorité ne me fait pas peur» - participait directement à la construction de l'éthos sarkozyste fait de courage (celui qui n'a pas peur, et va le prouver par la répétition d'un mot tabou), d'autorité ou de volonté.

9. Dans cet exemple, les «parce que » prennent encore une intonation interrogative, ailleurs ils sont clairement affirmatifs. "Parce que " est une spécificité lexicale très forte du discours de Sarkozy.

10. «La polémique est intrinsèque à l'emploi de ne... pas. [...] Il y a une seule négation ne... pas, et elle est polémique » (Nølke : 1992, 65-66).

11. N. Sarkozy, 3 mai, Montpellier. Cf. aussi : «Dans cette élection il s'agit de savoir si l'héritage de mai 68 doit être perpétué ou s'il doit être liquidé une bonne fois pour toutes » (N. Sarkozy, 29 avril 2007, Paris, Bercy).

12. Encore une fois, Nicolas Sarkozy explicite et annonce l'anaphore qu'il va produire : c'est à la suite du peuple qu'il va "répéter indéfiniment» cet "écho» d'exaspération. L'efficacité rhétorique de Sarkozy tient dans ces commentaires métalinguistiques ou mises en perspective de son discours. Ainsi lui arrive-t-il très souvent de gloser sur les mots qu'il emploie, de justifier les formules qu'il prononce, de souligner la figure qu'il produit.

13. L'exemple le plus caricatural fut l'introduction du meeting de la Concorde en 2012 où, sans autre explication, le candidat a déclaré : « Le peuple de France dit ça suffit ! (N. Sarkozy, 14 avril 2012, Paris). Sur le «ça » de Sarkozy, cf., outre le chapitre d'ouvrage cité (Mayaffre, 2012), D. Mayaffre (2014, 2013).

14. Pour plus d'effet encore, la continuité du discours, c'est-à-dire le passage d'une anaphore à l'autre (identité de la figure) autour d'un thème unique (identité du signifié) est assurée par une quasi anadiplose (cf. note en conclusion) : « La France c'est le pays... La France c'est le pays... C'est le pays qui... C'est le pays qui... ».

15. Mesurons par exemple que le fameux « I have a dream » fut répété 8 fois... soit 38 fois de moins que « pourquoi tant de haine ? » ou 18 fois de moins que « je veux être le président qui... », etc. 16. L'anadiplose est la répétition au début d'une unité syntaxique de l'élément qui avait conclu l'unité syntaxique précédente : «Il est bête. Bête il restera ».

\section{RÉSUMÉS}

Cette contribution décrit les innombrables - plusieurs milliers - anaphores rhétoriques du discours électoral de Nicolas Sarkozy, et essaye d'en déterminer les fonctions pragmatiques. Une typologie des anaphores est proposée avec une attention particulière apportée aux anaphores complexes (anaphores filées, épiphores, anaphores d'anaphores) et une interprétation 
pragmatique est donnée de la récurrence de la figure: par la répétition volontaire, Sarkozy construit l'ethos d'un chef charismatique fier et sûr de ses convictions.

This paper describes the myriads of rhetorical anaphora - there are several thousands of them in the campaign speech of Nicolas Sarkozy, and seeks to determine their pragmatic functions. This paper proposes a typology of anaphora with special emphasis on three types of complex anaphora (extended anaphora, epiphora, anaphora of anaphora). It provides the following pragmatic interpretation for the recurrence of the device: through voluntary repetition, Sarkozy builds the ethos of a charismatic leader who is sure and proud of his convictions.

INDEX

Keywords : rhetorical anaphora, epiphora, logometry, political discourse, ethos, Sarkozy

Mots-clés : anaphore rhétorique, épiphore, logométrie, discours politique, ethos, Sarkozy

\section{AUTEUR \\ DAMON MAYAFFRE}

BCL (UMR 7320), CNRS 